\title{
Broadband Doppler-limited two-photon and stepwise excitation spectroscopy with laser frequency combs
}

\author{
Arthur Hipke, ${ }^{1,2}$ Samuel A. Meek, ${ }^{2}$ Takuro Ideguchi, ${ }^{2}$ Theodor W. Hänsch, ${ }^{1,2}$ and Nathalie Picqué ${ }^{1,2,3, *}$ \\ ${ }^{1}$ Ludwig-Maximilians-Universität München, Fakultät für Physik, Schellingstraße 4/III, 80799 Munich, Germany \\ ${ }^{2}$ Max-Planck-Institut für Quantenoptik, Hans-Kopfermann-Straße 1, 85748 Garching, Germany \\ ${ }^{3}$ Institut des Sciences Moléculaires d'Orsay, CNRS, Bâtiment 350, Université Paris-Sud, 91405 Orsay, France
}

(Received 24 November 2013; published 21 July 2014)

\begin{abstract}
Multiplex two-photon excitation spectroscopy is demonstrated at Doppler-limited resolution. We describe first Fourier-transform two-photon spectroscopy of an atomic sample with two mode-locked laser oscillators in a dual-comb technique. Each transition is uniquely identified by the modulation imparted by the interfering comb excitations. The temporal modulation of the spontaneous two-photon fluorescence is monitored with a single photodetector, and the spectrum of all excited transitions is revealed by a Fourier transform.
\end{abstract}

DOI: 10.1103/PhysRevA.90.011805

Two-photon excitation is a widely exploited nonlinear phenomenon with applications that extend to the fields of spectroscopy, photochemistry, and biochemical imaging. In atomic and molecular spectroscopy, because two-photon selection rules are different from one-photon (electric dipole) selection rules, two-photon transitions may allow access to states which otherwise could not be reached. Furthermore, parts of the energy spectrum where laser radiation is hardly available may be explored. Most of the time, the experiments have been performed with tunable continuous-wave or pulsed lasers, leading to a wide variety of powerful experimental schemes and valuable spectroscopic data. The increased availability of ultrashort-pulse lasers has been the trigger for a fast uptake of the two-photon excitation technique in chemistry and biology, because they provide efficient excitation, although they usually do not allow for spectral discrimination. High resolution may be achieved [1-6] with a train of pulses from a modelocked laser, because the spectrum of an optical frequency comb is composed of narrow evenly spaced lines. Even the first experiments with a picosecond laser demonstrated [1,2] the potential of this technique for sub-Doppler resolution and direct spectral calibration. The resonance condition for excitation of a given atomic energy level can be satisfied by many pairs of comb lines, so that the excitation probability of the level can be the same as for a resonantly tuned continuous-wave laser of the same average power. However, in this type of direct frequency-comb spectroscopy, any energy level spacing can only be measured modulo the line spacing of the frequency comb. The technique is only suitable for very simple spectra with sharp transitions and is not appropriate for broad and crowded spectra or for transitions with a linewidth broader than the comb-line spacing, as often encountered with Doppler-broadened profiles. These spectroscopic techniques require the frequency of the laser(s) to be tuned to explore the spectrum. Multiplex or multichannel techniques, in which a broad spectral bandwidth is simultaneously recorded, are however advantageous, because they usually result in reduced experimental time and improved consistency of the spectral data.

\footnotetext{
*nathalie.picque@mpq.mpg.de
}

PACS number(s): 42.62.Fi, 33.20.-t, 33.80.Rv, 78.47.jh

To achieve multiplex detection which can disambiguate signals from different energy levels, it has been proposed to modulate the output of a mode-locked laser with a varying arm Michelson interferometer [7]. Subsequently, a few investigations in the liquid phase at low resolution $(>2$ $\mathrm{THz}$ ) took advantage [8-10] of Michelson-based two-photon Fourier-transform spectroscopy.

In this Letter, we describe a new technique of two-photon excitation spectroscopy. With two laser frequency combs, we are able to simultaneously acquire on a single photodetector a broad two-photon excitation spectrum of Doppler-broadened transitions. We first describe the general principle of the technique and we then present a proof-of-concept experiment on atomic rubidium vapor. Around $770 \mathrm{THz}$, spectra of the $5 S-5 D$ transitions with a Doppler width of the order of $1 \mathrm{GHz}$ are measured within tens of seconds, with a resolution up to $100 \mathrm{MHz}$.

In recent years, the exciting potential of dual-comb spectroscopy [11-15] for linear absorption measurements of Doppler-broadened transitions has been demonstrated. Two optical-frequency-comb generators of slightly different repetition frequencies produce - in the time domain — pairs of pulses (one from each comb) with a separation that changes linearly from pulse to pulse. The time-domain correlation function of the interfering combs, as filtered by the absorbing sample, is Fourier transformed to uncover the spectrum. The dual-comb approach thus mimics a scanning Michelson interferometer but, since there are no moving parts, the previous limitations in acquisition speed and resolution may be overcome. Because frequency combs involve intense femtosecond pulses, dualcomb spectroscopy can be extended to nonlinear phenomena, as recently demonstrated with coherent Raman effects [16]. Here we show how it can be harnessed for two-photon spectroscopy.

In dual-comb two-photon spectroscopy, two copropagating mode-locked femtosecond lasers with repetition frequencies $f_{\mathrm{r}}$ and $f_{\mathrm{r}}+\Delta f_{\mathrm{r}}$ drive two-photon transitions in an atomic or molecular sample. In the time domain, the excitation by pairs of pulses with a linearly increasing time separation produces Ramsey-like interference in the excitation amplitude. The population in the excited states depends on the competing processes of two-photon excitation, fluorescence decay, and possibly other processes such as radiationless decay. Its 


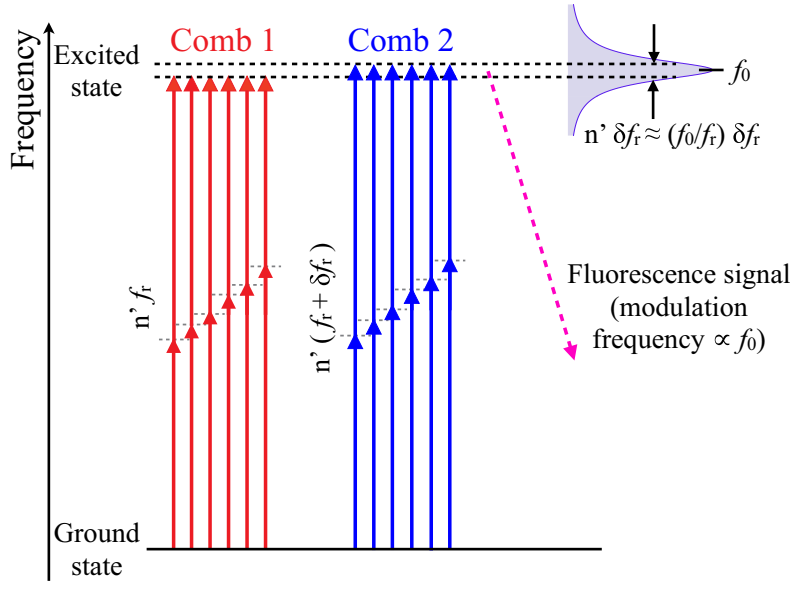

FIG. 1. (Color online) Frequency-domain illustration of the principle of dual-comb two-photon spectroscopy. For simplicity, we assume in this figure that the carrier-envelope offset frequency of the two frequency combs is equal to zero. Pairs of comb modes with the same sum frequency $\left[n^{\prime} f_{\mathrm{r}}\right.$ for comb $1, n^{\prime}\left(f_{\mathrm{r}}+\delta f_{\mathrm{r}}\right)$ for comb 2] resonantly excite a two-photon transition at the optical frequency $f_{0}$. The excitation rate of the transition is modulated at the beat frequency $n^{\prime} \delta f_{\mathrm{r}}$, which is approximately equal to $\frac{f_{0}}{f_{\mathrm{r}}} \delta f_{\mathrm{r}}$. An intensity modulation of frequency $\frac{f_{0}}{f_{\mathrm{r}}} \delta f_{\mathrm{r}}$ is thus observed in the fluorescence radiated during decays to lower states. Because the frequency of the modulation is proportional to the transition frequency $f_{0}$, the two-photon excitation spectrum is revealed by Fourier transformation of the fluorescence intensity recorded versus time.

modulations are recorded, as a function of time, via the fluorescence emitted during decays to lower states. The measured time-domain interference signal, the interferogram, is Fourier transformed to reveal the spectrum of the excited two-photon transitions.

In the frequency domain, each laser produces a comb of modes with evenly spaced optical frequencies. For instance, for comb 1, the optical frequencies $f_{n, 1}$ may be written $f_{n, 1}=n f_{r}+f_{\text {ceo, } 1}$, where $n$ is an integer and $f_{\text {ceo, } 1}$ is the carrier-envelope offset frequency. Many pairs of modes (Fig. 1) may satisfy the resonance condition for two-photon excitation at the same time. Possible sum frequencies for comb 1 are given by $f_{\text {sum }, 1}=n^{\prime} f_{\mathrm{r}}+2 f_{\text {ceo, } 1}$, where $n^{\prime}$ is integer valued. Two sum frequencies, $f_{\text {sum, } 1}$ for comb 1 and $f_{\text {sum, } 2}$ for comb 2, interfere and the two-photon excitation rate is modulated at the beat-note frequency $n^{\prime} \delta f_{\mathrm{r}}+2 \delta f_{\text {ceo }}$, where $\delta f_{\text {ceo }}$ is the difference of the carrier-envelope offset frequency of the two combs. The excited-state fluorescence, which may be monitored through some indirect decay channel, is therefore modulated at the beat-note frequencies within the excited line profile. As shown in Fig. 1, if a transition of optical frequency $f_{0}$ is excited, the intensity of the fluorescence is modulated at a frequency of about $\frac{f_{0}}{f_{\mathrm{r}}} \delta f_{\mathrm{r}}+2 \delta f_{\text {ceo. The }}$. The two-photon spectrum is thus mapped to radio-frequency beat notes that are directly proportional to the two-photon transition frequencies. The measurement of the repetition frequency and carrier-envelope offset frequency of the combs allows for converting the measured radio frequencies to the proper optical scale. We emphasize that, unlike in experiments using an external electro-optic modulator for frequency modulation
$[17,18]$, the signal is unequivocally encoded at frequencies proportional to the optical frequencies of the transitions. Therefore it is possible to simultaneously detect on a single photodetector all the excited transitions without tuning or filtering. This highly multiplexed capability is the main advantage of our technique.

For a first proof-of-principle experiment, we have chosen two-photon spectroscopy of the $5 S-5 D$ transitions in atomic rubidium vapor with a resonant $5 P_{3 / 2}$ intermediate state. The $5 P_{3 / 2}$ level can be resonantly excited by one photon and its excitation rate is modulated by the interference of the exciting comb lines. The resulting population pulsations can be detected in the fluorescence light after stepwise two-photon excitation. Thus the fluorescence light is modulated at three groups of beat frequencies: (i) $m \delta f_{\mathrm{r}}+\delta f_{\text {ceo }}$, corresponding to one-photon comb excitation from the ground to the intermediate state, (ii) $k \delta f_{\mathrm{r}}+\delta f_{\text {ceo }}$ corresponding to one-photon comb excitation from the intermediate to the final excited state, and (iii) $n^{\prime} \delta f_{\mathrm{r}}+2 \delta f_{\text {ceo }}$, corresponding to two-photon comb excitation from the ground to the final excited state. The Fourier transform of the interferogram reveals a spectrum mapping the one- and two-photon transitions in unequivocal regions.

The experimental setup is shown in Fig. 2. Two amplified erbium-doped fiber comb generators with a repetition frequency of about $100 \mathrm{MHz}$ emit pulses of a duration of the order of $100 \mathrm{fs}$. Their emission is centered around $192 \mathrm{THz}$ $(1.56 \mu \mathrm{m})$ and their average power is $150 \mathrm{~mW}$. To prevent long-term drifts, the repetition frequency of each comb is stabilized (not shown in Fig. 2) against a radio-frequency clock. This is achieved by adjusting the laser cavity length, because the cavity includes a free-space part with an end mirror mounted onto a piezoelectric transducer. One optical line of each comb is phase locked to a free-running continuous-wave erbium-doped fiber laser emitting at $192 \mathrm{THz}(1.56 \mu \mathrm{m})$ by feedback on the current of the pump diodes of the combs. To avoid aliasing in dual-comb spectroscopy, the frequency of all radio beat notes must be lower than half the comb repetition frequency. This determines how the repetition frequency difference $\delta f_{\mathrm{r}}$ should be set: $\delta f_{\mathrm{r}} \leqslant f_{\mathrm{r}}^{2} /(2 \Delta f)$ where $\Delta f$ is the bandwidth of the spectrum. Here an additional limitation

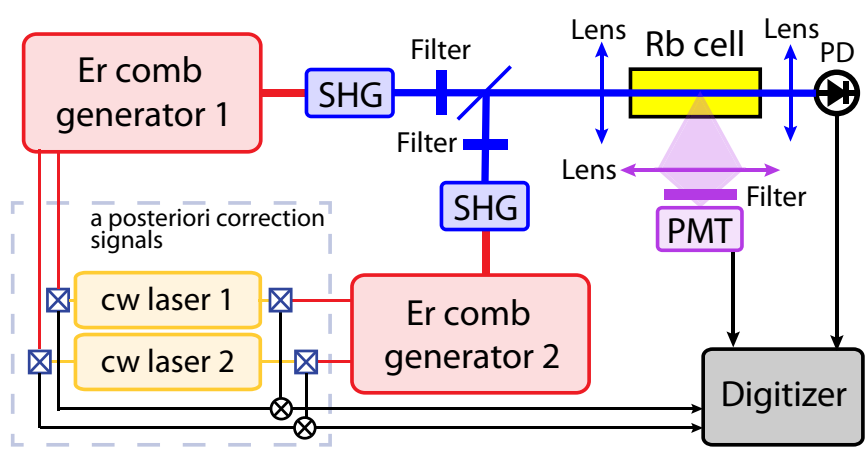

囚optical beat detection $\otimes$ electronic mixer

FIG. 2. (Color online) Experimental setup for dual-comb twophoton spectroscopy of atomic rubidium vapor. cw, continuous-wave; SHG, second-harmonic generation; PMT, photomultiplier tube; PD, photodiode. 
arises: the relatively long lifetime of the $5 D$ states (about $240 \mathrm{~ns}$ ) acts as a low-pass filter on the modulated fluorescence signal in the radio-frequency domain, limiting the maximum modulation frequency to a few times the natural linewidth; here, a few MHz. Such a filtering effect was already pointed out in frequency-modulation spectroscopy with an external modulator and is discussed in Ref. [18]. Therefore, we must choose a relatively low repetition-frequency difference (here, $\delta f_{\mathrm{r}}=1 \mathrm{~Hz}$ ). The outputs of the erbium comb generators are frequency doubled in magnesium-oxide-doped periodically poled lithium niobate (MgO:PPLN) crystals of a thickness of $0.5 \mathrm{~mm}$, yielding spectra centered around $383 \mathrm{THz}(783 \mathrm{~nm})$, with a full width at half maximum of about $10 \mathrm{THz}(20 \mathrm{~nm})$. Dichroic mirrors isolate the second-harmonic radiation. The two $383 \mathrm{THz}$ beams are then combined in a beam-splitter cube and focused into a heated 30-mm-long cell filled with rubidium in natural abundance. Each frequency-doubled comb has an average power of about $20 \mathrm{~mW}$ at the sample. We thus excite $\mathrm{Rb}$ into the $5 D$ states [Fig. 3(a)] with our dual-comb system. From the $5 D$ states, $\mathrm{Rb}$ atoms can decay into the $6 P$ manifold, from where they emit fluorescence at 711 and $713 \mathrm{THz}$ to repopulate the $5 S$ ground state. We collect the $6 P-5 S$ fluorescence of the sample through a side window, mostly in the region of the focal spot, to measure the interferograms. No particular care, however, has been taken to image or spatially filter this region. The fluorescence is spectrally filtered with an interference filter to reduce scattered light from the laser beam and is detected with a photomultiplier tube. The intensity of the fluorescence is modulated at all beat-note frequencies corresponding to the resonances excited by the combs. The one-photon and two-photon transitions, respectively, are imaged in the 0.64 and $1.28 \mathrm{MHz}$ radiofrequency regions, respectively. To avoid aliasing and excess noise while preserving the entire bandwidth in the spectra, an electronic low-pass filter (cutoff frequency: $1.9 \mathrm{MHz}$ ) is used. The electric signal is then digitized with a 14-bit analogto-digital converter at a sampling rate of $5 \times 10^{6}$ samples/s. In addition, the laser beams that are transmitted through the sample may beat on a fast Si photodiode, thus allowing for the simultaneous measurement of a dual-comb linear absorption interferogram.

As already discussed, e.g., in Ref. [14], stabilizing the combs to a radio-frequency reference involves low-bandwidth feedback loops that do not compensate for the short-term instabilities of the combs. Such instabilities severely distort the spectra. We therefore monitor the relative fluctuations of the two combs and apply an a posteriori correction algorithm similar to that reported in Ref. [15]. The beat signals between two pairs of individual comb lines of the two different combs in two different spectral regions are isolated with the aid of two auxiliary continuous-wave erbium-fiber lasers emitting at $192 \mathrm{THz}(1.56 \mu \mathrm{m})$ and $195 \mathrm{THz}(1.53 \mu \mathrm{m})$, respectively, as local oscillators. Electronic mixers cancel out any fluctuation of the local oscillators. The two resulting radio-frequency beat signals are recorded as a function of time simultaneously to the interferogram. They provide the necessary correction signals of the timing jitter and relative fluctuations of the carrier-envelope phase of the combs. They are used in a computer algorithm to multiply the interferogram by a time-varying phase factor and to subsequently correct for timing fluctuations by adjusting the sampling rate to an equidistant grid. The correction signals are derived from the outputs of the erbium femtosecond oscillators around $192 \mathrm{THz}$ (fundamental frequency). In order to properly correct the one-photon spectra that are centered around $385 \mathrm{THz}$ (second harmonic) and the two-photon spectra that are centered around $771 \mathrm{THz}$ (fourth harmonic), we multiply the interferometric phase by an appropriate order factor (two for the second harmonic, four for the fourth harmonic). We thus extend previous correction schemes [15] to dual-comb nonlinear spectroscopy and show that monitoring the relative instabilities of the oscillators at their fundamental frequency is effective for correction of interferograms involving nonlinear frequency generation and/or nonlinear phenomena. Figure 3(b) shows an a-posteriori-corrected interferogram of the modulations in the $6 P-5 S$ fluorescence of ${ }^{85} \mathrm{Rb}$ and ${ }^{87} \mathrm{Rb}$, which exhibits contrasted fringes over the entire range of the recording. The contribution of the two-photon transitions to the interferogram, (a)

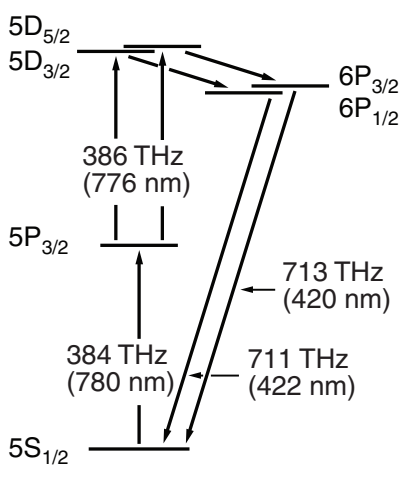

(b)

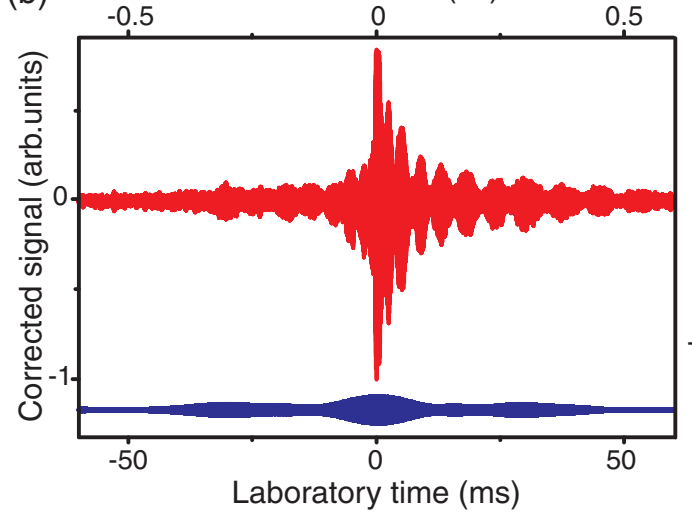

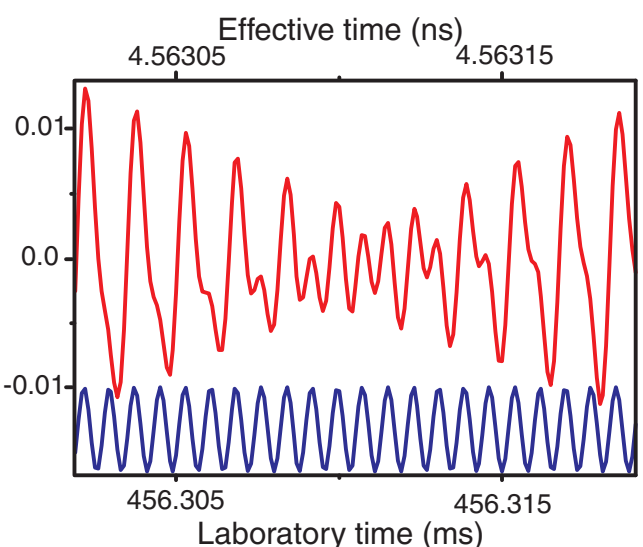

FIG. 3. (Color online) (a) Experimentally relevant fine structure of rubidium. (b) Two portions of an unaveraged, corrected interferogram recorded at $T=393 \mathrm{~K}$ with $\delta f_{r}=1 \mathrm{~Hz}$ (red) and contributions to that interferogram from two-photon transitions (blue). The two-photon interferometric traces (blue) have been vertically-shifted for clarity. Two time scales are displayed. The laboratory time is the actual measurement time which accounts for the down-sampling nature of the technique. The effective time is the time delay between two pulses in a pair, one pulse from each comb. 
(a)

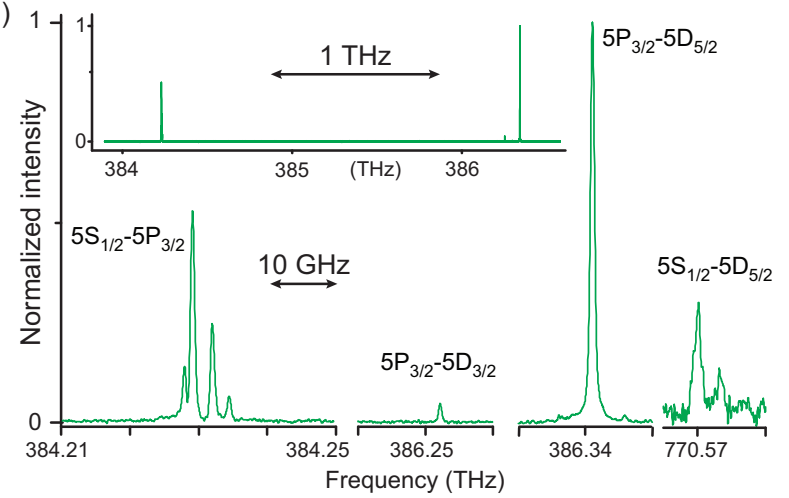

(b)

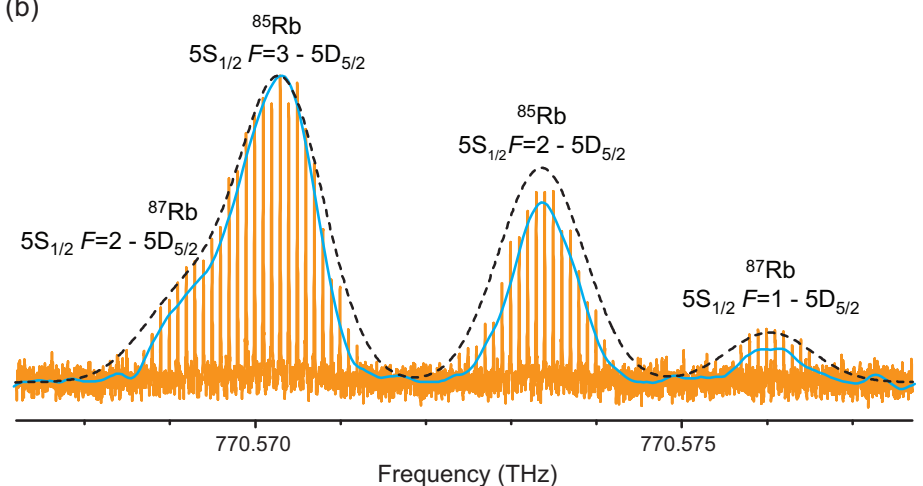

FIG. 4. (Color online) Experimental dual-comb spectra. (a) Portions of the one- and two-photon excitation spectrum of Rb. The entire range of the one-photon excitation lines is shown in the upper-left part of the panel, while the main part zooms in to the individual one- and two-photon transitions (tick increment is $10 \mathrm{GHz}$ ). The spectrum, displayed at an unapodized resolution of $300 \mathrm{MHz}$, is recorded at $T=308 \mathrm{~K}$ within $18 \mathrm{~s}$ measurement time. (b) Portion of the two-photon spectrum recorded at $T=393 \mathrm{~K}$ (solid cyan: $18 \mathrm{~s}$ measurement time, unapodized resolution of $300 \mathrm{MHz}$; solid orange: $54 \mathrm{~s}$ measurement time, unapodized comb transform-limited linewidth of $4.4 \mathrm{MHz}$; dashed black: computed).

frequency-filtered a posteriori by a computer program, is also shown.

Fourier transforming an interferogram reveals a one- and two-photon spectra [Figs. 4(a) and 4(b)]. Within the excitation range of our lasers, rubidium has [Fig. 3(a)] three one-photon resonances $\left(5 S_{1 / 2}-5 P_{3 / 2}, 5 P_{3 / 2}-5 D_{3 / 2}\right.$, and $\left.5 P_{3 / 2}-5 D_{5 / 2}\right)$ and two two-photon lines $\left(5 S_{1 / 2}-5 D_{3 / 2}\right.$ and $\left.5 S_{1 / 2}-5 D_{5 / 2}\right)$. The $5 S_{1 / 2}$ ground state of both isotopes is split [not shown in Fig. 3(a)] by a few $\mathrm{GHz}$ due to the hyperfine interaction. The hyperfine splitting of the $5 P$ and $5 D$ states is narrower than the Doppler linewidth (about $1 \mathrm{GHz}$ ) and is thus not resolved in our spectra. While the entire spectral range of excitation simultaneously spans from 373 to $393 \mathrm{THz}$ and from 755 to $780 \mathrm{THz}$ (corresponding to $1.17 \times 10^{5}$ spectral elements, as defined by the ratio of range of excitation to resolution), only four narrow frequency segments [Fig. 4(a)] exhibit spectral lines. Figure 4(b) displays the frequency segment centered around $770.572 \mathrm{THz}$ from a single interferogram, Fourier transformed at two different resolutions. Such an interferogram is recorded at high temperature (393 K), which is required to obtain a reasonable signal-to-noise ratio in the two-photon transitions. For a measurement duration of $54 \mathrm{~s}$, the spectrum [Fig. 4(b)] is composed of individual comb lines, showing that the coherence between the two mode-locked lasers is reconstructed. The comb-line spacing of $100 \mathrm{MHz}$ in Fig. 4(b) represents the best resolution that can be achieved in such measurements. The spectra are calibrated by using the measured repetition frequencies of the combs and one of the $\mathrm{Rb}$ lines. The spectrum of Fig. 4(a) is calibrated against the ${ }^{85} \mathrm{Rb}$ $5 S_{1 / 2}, F=2-5 P_{3 / 2}$ transition [19,20], whereas the spectra of Fig. $4\left(\right.$ b) are calibrated against the ${ }^{85} \mathrm{Rb} 5 S_{1 / 2}, F=2-5 D_{5 / 2}$ transition [21]. The other line positions then agree within 40 $\mathrm{MHz}$ with the centroid values derived from Refs. [19-21]. The two-photon lines are correctly reproduced by calculations that incorporate all allowed hyperfine transitions and assume Doppler profiles. Simultaneously to the fluorescence interferogram, we acquire the linear-absorption interferogram. A portion of the resulting spectrum (at $308 \mathrm{~K}$ ) is shown in Fig. 5. This adds to our technique an additional diagnostic tool, which might prove useful if the sample is not spectroscopically well known.

The above proof-of-principle demonstration shows the potential of dual-comb spectroscopy for the measurement of broad-spectral-bandwidth Doppler-limited two-photon excitation spectra. The technique applies to any sample that fluoresces under two-photon excitation and could be extended to multiphoton fluorescence or ionization. It permits highly multiplexed measurements since all one- and two-photon transitions within the excitation bandwidth of the femtosecond lasers are simultaneously observed within a short measurement time. Background-free fluorescence detection allows for higher sensitivity than in absorption measurements. Dualcomb two-photon spectroscopy could readily be applied for spatially selective real-time spectroscopy of liquid samples. As only a single photodetector is needed, it may easily be implemented in existing two-photon microscopes for hyperspectral mapping. In these applications, with the same experimental setup, dual-comb two-photon spectroscopy can be complemented with, e.g., dual-comb coherent anti-Stokes Raman spectroscopy [16] to simultaneously interrogate vibronic and vibrational transitions. Moreover, if the two laser combs both counterpropagate in the sample, the technique can be extended to sub-Doppler resolution and hence to the field of precision

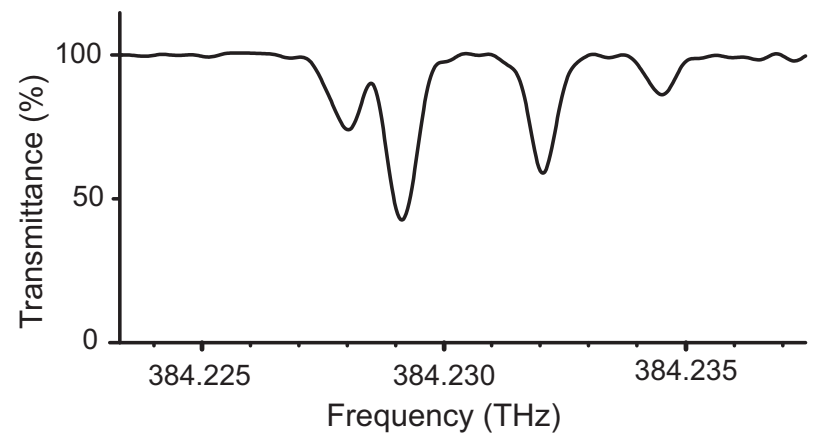

FIG. 5. Portion of the absorption dual-comb spectrum of $\mathrm{Rb}$ at $T=308 \mathrm{~K}$ and $300 \mathrm{MHz}$ unapodized resolution. 
spectroscopy. Preliminary results [22] indicate the feasibility of such experiments with conditions similar to those reported in this Letter. In contrast to direct frequency-comb two-photon spectroscopy with a single mode-locked laser, the optical free spectral range in dual-comb two-photon spectroscopy has no other limit than the spectral bandwidth of the laser frequency combs. Thus Doppler-free spectroscopy of highly crowded molecular spectra should become feasible.

\section{ACKNOWLEDGMENTS}

Experimental support from Birgitta Bernhardt and Antonin Poisson is warmly acknowledged. Support by the European Research Council (Advanced Investigator Grant 267854), the Max Planck Foundation, the European Laboratory for Frequency Comb Spectroscopy, and the Munich Center for Advanced Photonics is acknowledged.
[1] R. Teets, J. Eckstein, and T. W. Hänsch, Phys. Rev. Lett. 38, 760 (1977).

[2] J. N. Eckstein, A. I. Ferguson, and T. W. Hänsch, Phys. Rev. Lett. 40, 847 (1978).

[3] A. Marian, M. C. Stowe, J. R. Lawall, D. Felinto, and J. Ye, Science 306, 2063 (2004).

[4] J. E. Stalnaker, V. Mbele, V. Gerginov, T. M. Fortier, S. A. Diddams, L. Hollberg, and C. E. Tanner, Phys. Rev. A 81, 043840 (2010).

[5] I. Barmes, S. Witte, and K. S. E. Eikema, Phys. Rev. Lett. 111, 023007 (2013).

[6] E. Peters, D. C. Yost, A. Matveev, T. W. Hänsch, and T. Udem, Ann. Phys. (Berlin, Ger.) 525, L29 (2013).

[7] M. Bellini, A. Bartoli, and T. W. Hänsch, Opt. Lett. 22, 540 (1997).

[8] J. P. Ogilvie, K. J. Kubarych, A. Alexandrou, and M. Joffre, Opt. Lett. 30, 911 (2005).

[9] K. Isobe, A. Suda, M. Tanaka, F. Kannari, H. Kawano, H. Mizuno, A. Miyawaki, and K. Midorikawa, Phys. Rev. A 77, 063832 (2008).

[10] H. Hashimoto, K. Isobe, A. Suda, F. Kannari, H. Kawano, H. Mizuno, A. Miyawaki, and K. Midorikawa, Appl. Opt. 49, 3323 (2010).

[11] B. Bernhardt, A. Ozawa, P. Jacquet, M. Jacquey, Y. Kobayashi, T. Udem, R. Holzwarth, G. Guelachvili, T. W. Hänsch, and N. Picqué, Nat. Photonics 4, 55 (2010).
[12] I. Coddington, W. C. Swann, and N. R. Newbury, Phys. Rev. Lett. 100, 013902 (2008).

[13] F. Keilmann, C. Gohle, and R. Holzwarth, Opt. Lett. 29, 1542 (2004).

[14] T. Ideguchi, A. Poisson, G. Guelachvili, N. Picqué, and T. W. Hänsch, Nat. Commun. 5, 3375 (2014).

[15] J.-D. Deschênes, P. Giaccari, and J. Genest, Opt. Express 18, 23358 (2010).

[16] T. Ideguchi, S. Holzner, B. Bernhardt, G. Guelachvili, N. Picqué, and T. W. Hänsch, Nature (London) 502, 355 (2013).

[17] W. Lenth, C. Ortiz, and G. Bjorklund, Opt. Commun. 41, 369 (1982).

[18] J. Snadden, R. Clarke, and E. Riis, Opt. Commun. 152, 283 (1998).

[19] U. Rapol, A. Krishna, and V. Natarajan, Eur. Phys. J. D 23, 185 (2003).

[20] A. Banerjee, D. Das, and V. Natarajan, Europhys. Lett. 65, 172 (2004)

[21] F. Nez, F. Biraben, R. Felder, and Y. Millerioux, Opt. Commun. 102, 432 (1993).

[22] A. Hipke, S. A. Meek, G. Guelachvili, T. W. Hänsch, and N. Picqué, in CLEO: 2013 Postdeadline, OSA Postdeadline Paper Digest (Optical Society of America, Washington, DC, 2013), paper CTh5C.8. 